\title{
Orbital wall restoration with primary bone fragments in complex orbital fractures: A preliminary study
}

\author{
Joo Sung Jung, \\ Dong Hee Kang, \\ Nam Kyu Lim, \\ Hyonsurk Kim
}

Department of Plastic and

Reconstructive Surgery, Dankook

University Hospital, Cheonan, Korea
Background: We have reported orbital wall restoration surgery with primary orbital wall fragment in pure blowout fractures using a combination of transorbital and transnasal approach in pure blow out fractures. However, this method was thought to be difficult to use for complex orbital wall fractures, since the sharp screw tip that fixate the maxillary wall increases the risk of balloon ruptures. In this study, we reviewed 23 cases of complex orbital fractures that underwent orbital wall restoration surgery with primary orbital wall fragment and evaluated the result.

Methods: A retrospective review was conducted of 23 patients with complex orbital fracture who underwent orbital restoration surgery with primary orbital wall fragments between 2012 and 2019. The patients underwent orbital wall restoration surgery with primary orbital wall fragment with temporary balloon support. The surgical results were evaluated by the Naugle scale and a comparison of preoperative and postoperative orbital volume ratio. Complex fracture type, type of screw used for fixation and complications such as balloon rupture were also investigated.

Results: There were 23 patients with complex orbital fracture that used transnasal balloon technique for restoration. 17 cases had a successful outcome with no complications, three patients had postoperative balloon rupture, two patients had soft-tissue infection, and one patient had balloon malposition.

Conclusion: The orbital wall restoration technique with temporary balloon support can produce favorable results when done correctly even in complex orbital wall fracture. Seventeen cases had favorable results, six cases had postoperative complications thus additional procedure seems necessary to complement this method.

Keywords: Enophthalmos / Orbital fractures / Zygomatic fractures

\section{INTRODUCTION}

The goal of orbital reconstruction is to restore ocular motility and to prevent enophthalmos by restoring the native orbital anatomy and volume, but operations on this kind of complex orbital fracture are technically demanding and pose a major challenge to orbital surgeons. Complex orbital fractures are an impure type of orbital fracture, as they involve not only the or-

\section{Correspondence: Dong Hee Kang}

Department of Plastic and Reconstructive Surgery, Dankook University Hospital, 201 Manghyang-ro, Dongnam-gu, Cheonan 31116, Korea

E-mail: dhkcool@daum.net

Received March 20, 2020 / Revised May 7, 2020 / Accepted June 12, 2020 bital walls, but also the orbital rim and adjacent structures, as seen in frontal, maxillary, and zygomatic fractures. Patients with these complex fractures require simultaneous reconstruction of the displaced orbital rim and mid-face buttresses and restoration of the orbital shape [1,2].

Most surgeons struggle to restore the shape of the orbit, since the orbital wall is so thin that it cannot be fixed firmly to its primary position, unlike mid-face buttresses. Therefore, it has become more common to reconstruct orbital wall fractures using various kinds of synthetic implants [3]. Although these implants are strong enough to maintain their shape and position in the orbital cavity, reconstructive surgery using implants has 
some drawbacks [2]. The author has reported an orbital wall restoration technique in which primary orbital wall fragment restored to its previous position and temporary extraorbital support is maintained with an inflated Foley catheter balloon in the maxillary sinus $[1,2]$. However, this orbital wall restoration method may be difficult to use for complex orbital wall fractures, since the sharp screw tip used for buttress fixation increases the risk of balloon rupture [4].

In light of this potential issue, we theorized that we could avoid balloon rupture by fixing the screw at the margin of the buttress, which would yield successful outcomes in complex orbital wall fractures. In this paper, we evaluated 23 patients who underwent complex orbital wall restoration using primary bone fragments with an uneventful postoperative course and favorable outcomes.

\section{METHODS}

\section{Subjects}

This retrospective study analyzed patients who underwent reconstruction of complex orbital wall fractures between 2012 and 2019.

\section{Ophthalmic examination}

Ophthalmic examinations for diplopia, extraocular muscle movement, and enophthalmos were done before surgery. Enophthalmos was measured using a Naugle exophthalmometer (Inami Inc., Tokyo, Japan) by measuring from the superior and inferior orbital rim to the apex of the cornea. Preoperative values were evaluated 1 day before surgery and follow-up was performed 6 months after treatment when atrophy and scarring of the orbital tissue were complete. All patients underwent computed tomography (CT) scans within 1 week after trauma and at 6 months after surgery. The volume of the orbit was calculated by applying integral calculus to the CT images. The orbital volume ratio (OVR) was obtained by comparing the volume of the injured orbit to that of the uninjured orbit.

\section{Surgical techniques}

After performing the forced duction test, open reduction of complex fractures was performed through standard approaches, such as the buccal sulcus, lateral eyebrow, and transconjunctival approaches.

Screws are usually fixed along the edges of the maxillary sinus, corresponding to vertical and horizontal facial buttresses such as inferior orbital rim, pterygomaxillary buttress and nasomaxillary buttress. After screw fixation, usually in the vertical and transverse facial buttresses to obtain adequate skeletal support, dissection was done until the fracture of the orbital floor was exposed. The orbital content herniated through the defect was repositioned and a curved Freer elevator was inserted transnasally into the maxillary ostium to restore the primary bone fragment of the fractured inferior orbital wall.

A 14-Fr or 16-Fr Foley catheter (Sewoon Medical Co., Ltd., Seoul, Korea) was then inserted through the maxillary ostium into the maxillary sinus. The Foley catheter balloon was inflated using saline solution mixed with radiopaque dye (Telebrix 30; Guerbet, Villepinte, France) until the orbital floor was restored with the primary bone fragment. Because the Foley catheter balloon placed within the sinus does not intersect with the facial buttress margin, the balloon is likely to avoid rupture caused by fixation screws (Fig. 1). Bony restoration of the orbit-
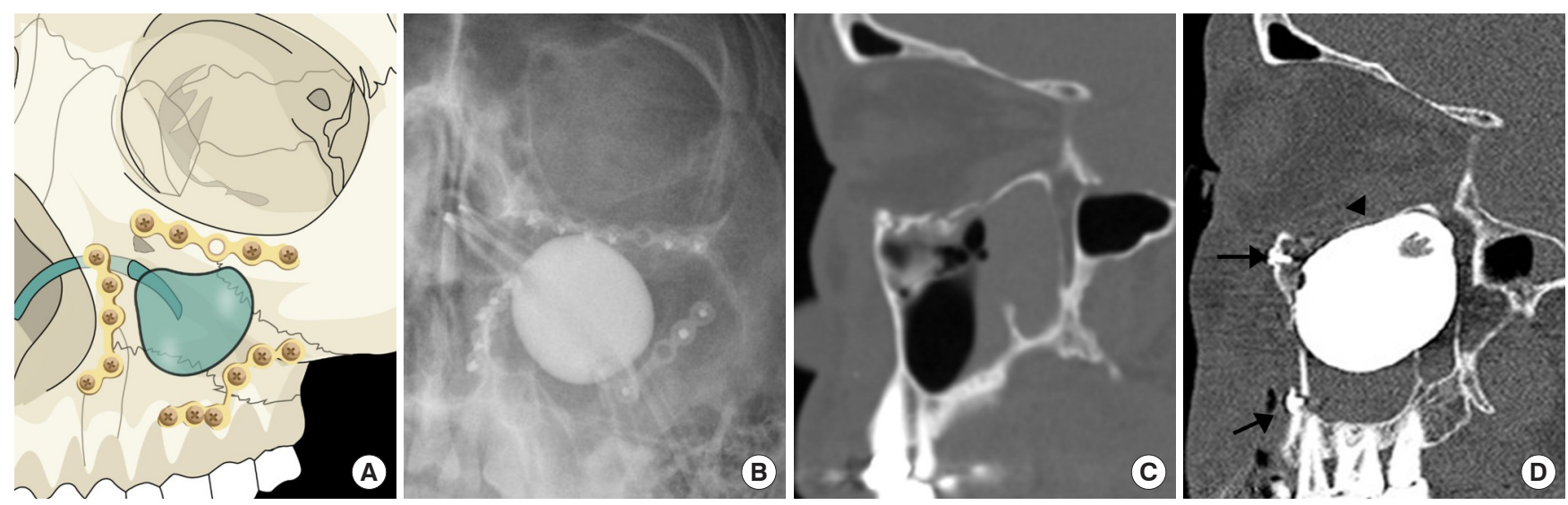

Fig. 1. When treating complex orbital wall fractures, a ballooned Foley catheter is placed within the pyramidal-shaped maxillary sinus. (A) An illustration of metal screws fixed in the inferior orbital rim, pterygomaxillary buttress and nasomaxillary buttress, which keeps the balloon from risk of rupture. (B) Postoperative X-ray. (C) Preoperative computed tomography (CT) scan. (D) Postoperative CT scan of a restored inferior orbital wall fracture with extraorbital support (black arrowhead) and reduction screws that do not touch the ballooned Foley catheter due to the space existing in between (black arrows). 
al floor was followed by placing a porous polyethylene implant such as Synpor (Synthes Inc., West Chester, PA, USA) or Medpor (Porex Surgical Inc., Newnan, GA, USA) or a resorbable mesh plate such as Rapidsorb (Synthes Inc.) to bridge the remaining defect [5]. The balloon support was kept in position for $7-8$ days.

\section{Statistical analysis}

The $t$-test was used to analyze perioperative differences in OVR and the Naugle scale. SPSS version 20.0 for Windows (IBM Corp., Armonk, NY, USA) was utilized for all analyses.

\section{RESULTS}

In total, 23 patients were treated with the standard open reduction method using the transnasal and transorbital approaches. Twenty-one patients were men and two were women (age range, 25-61 years; mean age, 38.6 years). The most common complex fracture type was zygomaticomaxillary complex fracture (14 cases), followed by nasomaxillary fracture (four cases), LeFort fracture (three cases), and maxillary fracture (two cases) (Table 1). The patients' postoperative course was mostly successful, with a short-term complication rate of $26.0 \%$. Shortterm complications included postoperative balloon rupture in three patients, soft-tissue infections in two patients, and balloon malpositioning in one patient (Table 2). Ruptured and malpositioned balloon cases were managed by removing the inserted balloon. Intravenous antibiotics were used to control soft-tissue infection patients which resolved within a week. All patients had a follow-up period of at least 6 months and showed favorable long-term outcomes (Fig. 2). The Naugle scale improved by an average of $0.27 \mathrm{~mm}$ and the OVR decreased by $7.05 \%$ on average (Table 3).

\section{DISCUSSION}

Historically, extraorbital support using a balloon was first reported in 1944 by Johnson, and cases where a Foley catheter was used for reduction of the anterior maxillary wall were first dealt with in 1975 by Oppenheimer [4,6]. Using the transnasal approach and temporary extraorbital support to restore the orbital floor was found to reduce the risk of volume change, postoperative implant shifting, and the size of the implant necessary for orbital wall restoration $[1,2]$.

The maxillary sinus is pyramidal in shape formed of four walls; anterior, posterior, superior and medial. The superior wall is made up of the fragile orbital floor and the medial wall separates the sinus from the nasal cavity. It has the maxillary ostium located between the middle and posterior thirds of the ethmoidal infundibulum and tend to be positioned closer to the roof of the sinus than the floor. The author inserted a Foley catheter through the maxillary ostium to restorate the orbital floor from the maxillary sinus. In complex orbital wall fractures, it is necessary to fixate the mid-face buttress fracture with plates and screws, which may increase the risk of the balloon rupture due to the sharp screw tip. Contrary to the common belief that Foley balloons have a high risk of rupture when used in complex orbital wall fractures [4], the authors could reduce the balloon rupture rate to $13.0 \%$ (3/23 cases).

The transnasal balloon method is beneficial because it gives more support to the restored primary orbital wall fragment, though there is risk of rupture in some cases. When the roundshaped Foley balloon is inflated in the sinus, the balloon supports the center areas of each sinus wall of the pyramid, but there is space left at the edge of the maxillary sinus. Fixing the screw to the edge of the maxillary buttress can reduce the balloon rupture rate by preventing the screw tip from touching the balloon. We used short screws $(<6 \mathrm{~mm})$ to prevent the screws from touching the balloon, in addition, absorbable packing

Table 2. Postoperative complications

\begin{tabular}{lc}
\hline Complication type & No. of cases (\%) \\
\hline Rupture of balloon & $3(13.0)$ \\
Soft-tissue infection & $2(8.6)$ \\
Malposition & $1(4.3)$ \\
Total & $6(26.0)$ \\
\hline
\end{tabular}

Table 1. Clinical data on 23 patients with complex orbital wall fractures

\begin{tabular}{|c|c|c|c|c|}
\hline \multirow{2}{*}{ Complex fracture type } & \multirow{2}{*}{ No. of case } & \multirow{2}{*}{ No. of complication } & \multicolumn{2}{|c|}{ Type of screw } \\
\hline & & & Metal & Resorbable \\
\hline Zygomaticomaxillary complex fracture & $14(60.8)$ & $2(8.6)$ & $14(60.8)$ & 0 \\
\hline Maxillary fracture & $2(8.6)$ & 0 & $1(4.3)$ & $1(4.3)$ \\
\hline Nasomaxillary fracture & $4(17.3)$ & $1(4.3)$ & $4(17.3)$ & 0 \\
\hline LeFort fracture & $3(13.0)$ & $3(13.0)$ & $3(13.0)$ & 0 \\
\hline Total & $23(100)$ & $6(26.0)$ & $22(95.6)$ & $1(4.3)$ \\
\hline
\end{tabular}

Values are presented as number (\%). 

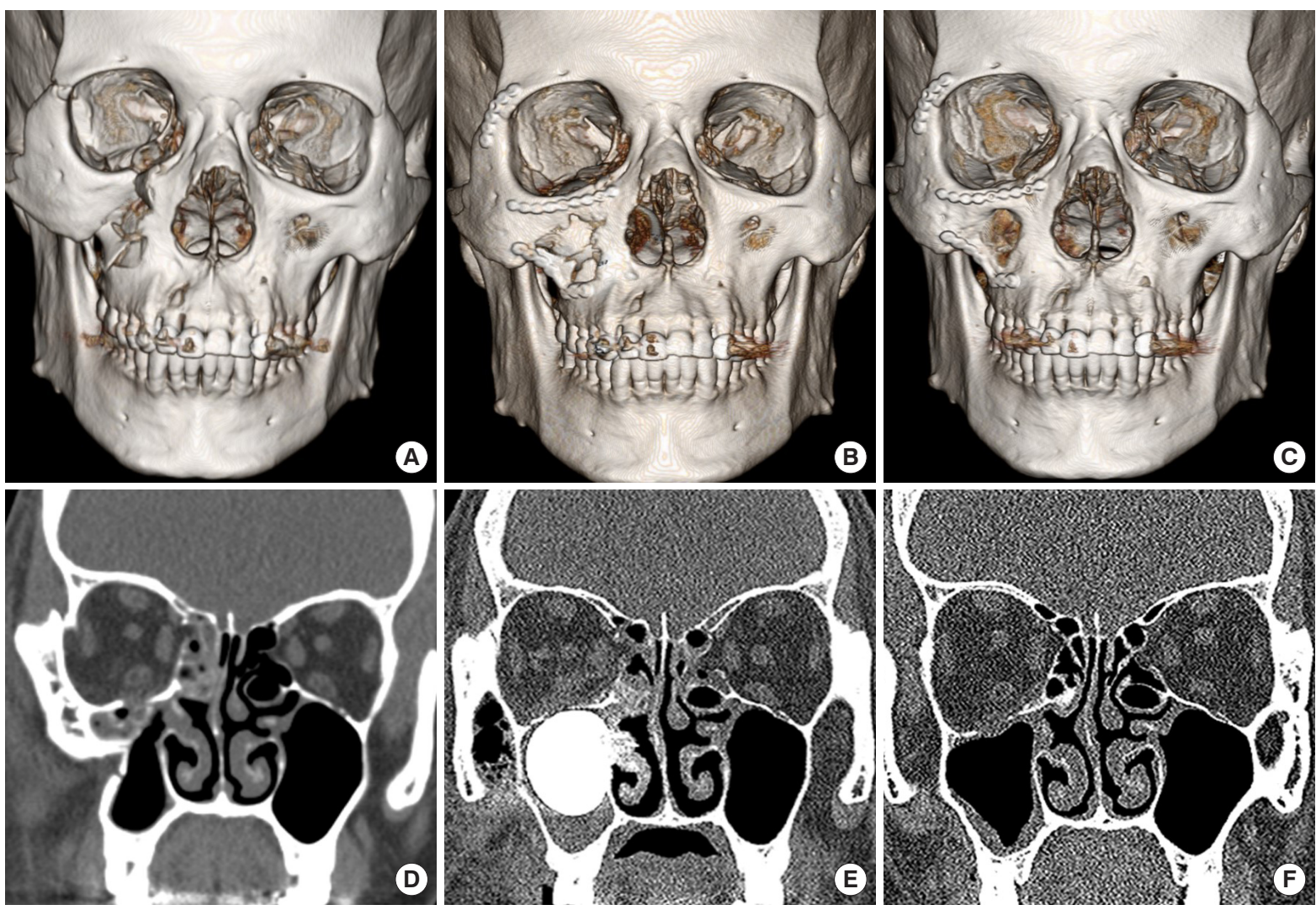

Fig. 2. Orbital wall restoration with primary orbital wall fragments using a balloon support was performed in cases of complex orbital wall fractures. The balloon provided a temporary balloon support to the restored orbital floor, avoiding the screws that were fixed in the vertical and horizontal facial buttresses. (A, D) Preoperative computed tomography (CT) scans. (B, E) Immediate postoperative CT scans of complex orbital wall fractures with screw fixation in facial buttresses, safe from balloon rupture. (C, F) Seven-month postoperative CT scans.

Table 3. Naugle exophthalmometry and orbital volume ratio in 23 patients with complex orbital wall fractures

\begin{tabular}{|c|c|c|c|c|c|c|c|}
\hline \multirow{3}{*}{ No. of cases } & \multicolumn{2}{|c|}{ Naugle exophthalmometry } & \multicolumn{5}{|c|}{ Orbital volume ratio (\%) } \\
\hline & \multirow{2}{*}{$\begin{array}{c}\Delta \text { Scale } \\
\text { (pre/post, mm) }\end{array}$} & \multirow{2}{*}{$p$-value ${ }^{a}$} & \multirow{2}{*}{ Unaffected orbit } & \multicolumn{2}{|c|}{ Affected orbit } & \multirow{2}{*}{ Volume change } & \multirow{2}{*}{$p$-value } \\
\hline & & & & Preoperative & Postoperative & & \\
\hline 23 & $0.27(0.85 / 1.12)$ & 0.63 & 100 & 109.97 & 102.92 & -7.05 & 0.28 \\
\hline
\end{tabular}

Scale $=$ affected globe position-unaffected globe position; $\Delta$ Scale= postoperative scale-preoperative scale.

a)Paired $t$-test.

sponges such as Nasopore (Polyganics B.V., Groningen, Netherlands) could be inserted through the maxillary ostium to protect the Foley balloon from rupture. In our cases, balloon rupture was found in three of the 23 cases, but two occurred 4 to 5 days after surgery and only one occurred one day after surgery. Although balloon rupture occurs at a low rate, if a balloon rupture by the screw is expected, it may be necessary to use a relatively large implant in complex orbital wall fracture.

It is recommended that investigations with larger sample sizes be performed to obtain more information on the correlations between the complex fracture type and the complication rate. In conclusion, our surgical results from 23 patients with complex orbital wall fractures suggest that providing additional sup- port from the maxillary sinus can yield good results and that this technique can be implemented effectively without significant risk of Foley catheter balloon rupture.

\section{NOTES}

\section{Conflict of interest}

No potential conflict of interest relevant to this article was reported.

\section{Ethical approval}

The study was approved by the Institutional Review Board of Dankook University Hospital (IRB No. DKUH 2020-06-011) 
and performed in accordance with the principles of the Declaration of Helsinki.

\section{ORCID}

Joo Sung Jung ～https://orcid.org/0000-0002-2515-9275

Dong Hee Kang https://orcid.org/0000-0002-6673-9655

Nam Kyu Lim ～https://orcid.org/0000-0002-0964-983X

Hyonsurk Kim https://orcid.org/0000-0001-6576-8035

\section{REFERENCE}

1. Kang DH. Orbital wall restoring surgery with primary orbital wall fragments in blowout fracture. Arch Craniofac Surg 2019; 20:347-53.

2. Lim NK, Kang DH, Oh SA, Gu JH. Orbital wall restoring surgery in pure blowout fractures. Arch Plast Surg 2014;41:686-
92.

3. Kakibuchi M, Fukazawa K, Fukuda K, Yamada N, Matsuda K, Kawai K, et al. Combination of transconjunctival and endonasal-transantral approach in the repair of blowout fractures involving the orbital floor. Br J Plast Surg 2004;57:37-44.

4. Hong SO, Lee DW, Lee JW, Jung JH. Minimal invasive and cost-effective method in reduction of maxillofacial fracture by using a Foley catheter ballooning technique. J Craniofac Surg 2013;24:e585-8.

5. Ramesh S, Hubschman S, Goldberg R. Resorbable implants for orbital fractures: a systematic review. Ann Plast Surg 2018;81: 372-9.

6. Park CH, Lee JH, Hong SM, Lee OJ. Reduction of inferior orbital wall fractures using a Foley catheter and an Endoloop. J Trauma 2011;70:E38-41. 